\title{
The MSOCC Data Switch Replacement: A Case Study in Elicitating and Elucidating Requirements
}

\author{
Joseph E. Kasser DSc. CEng, CM. \\ Systems Engineering and Evaluation \\ Centre \\ University of South Australia \\ Mawson Lakes, SA. 5095 \\ Joseph.Kasser@unisa.edu.au
}

Chandru J. Mirchandani CM

Lockheed Martin Transportation \&

Security Solutions, 9211 Corporate Boulevard,

Rockville, MD 20850.

Chandru.J.Mirchandani@1mco.com

\begin{abstract}
${ }^{1}$ Abstract. This paper is a case study of a situation in which a soft-systems methodology coupled with an object-oriented approach for viewing the requirements was used in a complex environment to gather a set of requirements. By considering the cost, priority, and risk attributes of the requirements, as well as clarifying the wording of the requirements for verifiability, an optimal systems architecture and development process was achieved in a relatively short period of time compared with the standard systems engineering process. Moreover, the Systems Requirements Review was deemed complete and comprehensive by the customer.
\end{abstract}

\section{Introduction ${ }^{2}$}

In 1989, the National Aeronautics and Space Agency's (NASA) Goddard Space Flight Center (GSFC) Multi-Satellite Operations Control Center (MSOCC) was facing the problem of replacing the data switch that routed signals from multiple low earth orbit (LEO) satellites to data processing computers. The problem was compounded by the following conditions:

- There was no physical space to locate a replacement switch in the MSOCC;

- The data streams from the satellites could not be switched off;

- Data could arrive at any time without warning;

- Loss of LEO satellite scientific data could not be tolerated;

- There were a plurality of stakeholders in the MSOCC

This paper describes how, by the use of a soft system intervention methodology, the operation and transition requirements for the data switch were developed to satisfy all stakeholders to the point where a complete consensus was documented at the data switch System Requirements Review (SRR) (NASA/GSFC, 1989b).

\section{Background}

At that time, the MSOCC was the major interface between the LEO data streams from the global satellite tracking network and the Telemetry Tracking and Control system at NASA's GSFC. There was minimal data capture and storage functionality in the ground stations and the network. The MSOCC was supported by two somewhat overlapping

\footnotetext{
${ }^{1}$ Copyright $\left({ }^{\circ} 2005\right.$ by Joseph Kasser. Published and used by INCOSE with permission.

${ }^{2}$ A table of Acronyms is located at the end of the paper.
} 
The 15th International Symposium of the International Council on Systems Engineering (INCOSE),

contracts, the Systems Engineering and Services (SEAS) contract and the Network Maintenance and Operations Support (NMOS) contract. The authors were with the SEAS subcontractor and were based in a location several miles from the GSFC. The SEAS contract was a cost-plus-award-fee (CPAF) type of contract.

The data streams from the LEO satellites contained data telemetered from onboard experiments and instruments. These data were supplied to Principal Investigators (PI) who would be very upset if they lost scientific data during the time period that the data switch was in transition. It was thus not acceptable to close down the MSOCC during the replacement of the data switch.

Technical details. The MSOCC used a switching system to route $1.544 \mathrm{MHz}$ serial asynchronous RS-422A digital data between the NASA Communication Network (NASCOM) and the computer equipment within MSOCC as well to rout the data within the MSOCC as shown below.

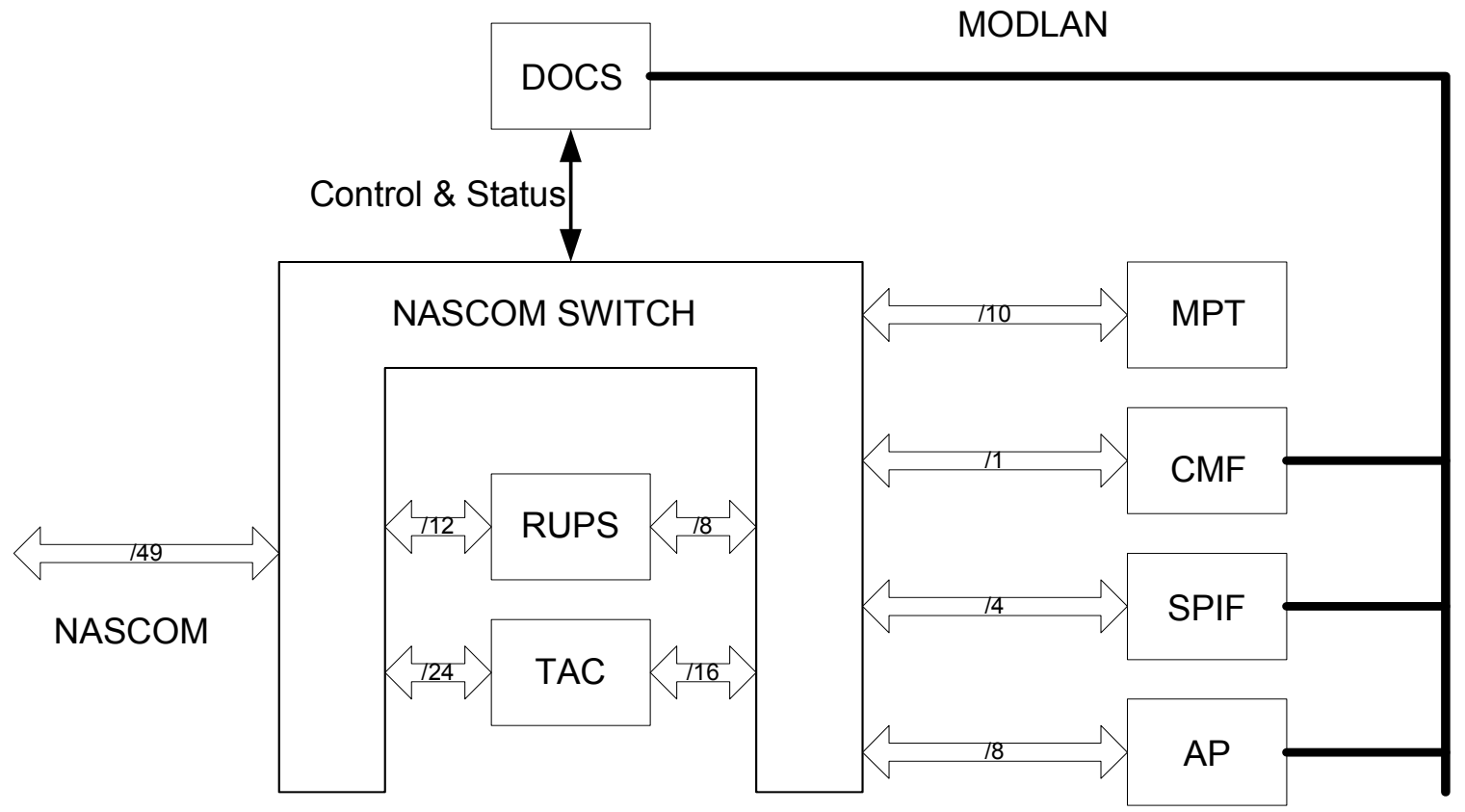

The NASCOM Switch shown as a single entity, really consisted of a number of subsystems including three separate switches controlled by a central Data Operations Control System (DOCS). The first switch connected some of the MSOCC equipment to the NASCOM and the second the remainder. The third switch handled connections between the Mission Planning Terminal (MPT), the Command Management Facility (CMF), the Deep Space Network (DSN), NASCOM and the Attached Shuttle Payload Center (ASPC). Each switch also contained a patch panel to allow the NASCOM lines to be tested, patched to another circuit, or looped back to NASCOM or MSOCC equipment.

To complicate the situation, the MSOCC forward link equipment sourcing uplink data to the LEO spacecraft did not generate the Send Timing (ST) signals (synchronizing pulses) to accompany the data. As a result, ST for this data was generated by a timing signal generator called a Clock Buffer located in each switch. 
The 15th International Symposium of the International Council on Systems Engineering (INCOSE),

The three switches were identical, each having a capacity of 62 full duplex ports. The switches had been custom-designed for the MSOCC and were not commercially available. Each switch had been added to the MSOCC over time in an incremental upgrade manner as the requirements for additional communications ports exceeded the number of ports available at the time the upgrade took place. Crossovers were used to connect Switch numbers 1 and 2. Switch number 3 was independent of the other two. As a result of using ports for crossovers, only 112 duplex connections could be made through the first two switches.

The need for a new switching system for MSOCC was based on the following deficiencies of the then current system.

a. The existing system had reached its physical limit and could not be expanded. The interfacing of new equipment already in existence (multiple NASCOM interfaces for the CCS 3280 Application Processors (AP), Recorder Utility Processors (RUPS) and the then still being planned History Recording System (HRS) and Transportable Payload Operation Control Center (TPOCC) could not be accommodated on the existing switch. The ability to interconnect the switches was limited to three full duplex crossovers between two of the switches. The third switch lacked any interconnection to the two other switches. Adding crossovers to accommodate the third would have required too many connections.

b. The direction of the ST signal on the MSOCC Data Terminal Equipment (DTE) interface was reversed when compared to the direction of the Send Data (SD) signal.

c. The ST signal was delayed with respect to the SD signal by the time the waveform took to propagate from the Clock Buffer to the data source and back to the switch along the cable.

d. The current switches only supported RS-422A circuits

e. In order to perform an internal loop back in the MSOCC, a NASCOM line on the switch had to be patched to provide that loop back. This patch panel effectively removed the NASCOM line from service.

f. The local control panel, which was the back-up system for controlling the switches, was difficult and often confusing to operate. This panel was not able to display the full status of its associated switch in one operation.

g. Command monitoring had to be accomplished via the patch panel through a 10:1 attenuated monitor position.

As a result of these deficiencies the need for a single switch to replace the three switches was recognized. The replacement switch was to have at least twice the connection capability of the existing three switches combined. The new switch was to be configured to handle data and timing in the same direction for both uplink and downlink data, which would alleviate the current phase problem between data and timing. The switch was to be controlled by the DOCS, handle data at standard digital rates of between 1.544 and $6.312 \mathrm{Mbps}$, and have a capacity of 255 full-duplex connections. The new switch system was to be named the MSOCC Communications Switching System (MCSS). 
The 15th International Symposium of the International Council on Systems Engineering (INCOSE),

\section{The Requirements elicitation and elucidation process}

The requirements elicitation and elucidation took place in the following sequential tasks:

- Determination of lessons learned from past projects.

- Determination of stakeholders

- Determination of the areas of concern for the major stakeholder

- Identification of the operational scenarios for the MCSS

- Determination of requirements for the MCSS

- Pre-SRR architecting

- Identification of candidate switch architectures

- Identification of candidate transition approaches

- Determination of test methodology for the MCSS upgrade

- The Systems Requirements Review

Determination of lessons learned from past projects. Lacking access to any corporate or customer information, we reviewed personal experiences and the literature in the domain of Quality and Management and researched factors that resulted in effective and ineffective systems engineering, and determined to make the project a success. We speedily identified that clear and concise communications were the key to the success of a requirements elicitation and elucidation project.

Determination of stakeholders. We identified the stakeholders by determining who was involved in the operations and maintenance of the MSOCC and actively involved the NASA GSFC Associate Technical Representative (ATR) ${ }^{3}$ in the process of identification of stakeholders. We then determined the stakeholder concerns and their requirements for the MCSS. This necessitated arranging a number of meetings with the different groups of stakeholders at their offices at the GSFC. Since we were not located on the base, these meetings had to be formal and coordinated ahead of time. Stakeholders were asked to provide two categories of requirements, mandatory and "wishes". The "wish" category was one where if a decision had to be made to implement a mandatory requirement, and a "wish" could be implemented with little or no extra cost, the "wish" would be taken into account.

During the interviews of the stakeholders, the critical questions asked were:

- What is good about the current system?

- What is bad about the current system?

- What would you change, and why?

When the responses from the different stakeholders to the questions were compared, we found that some of the answers were complementary and some were contradictory. The plurality was resolved by weighting the responses based on the relative importance of the stakeholders and holding a second meeting with any stakeholder who had been overruled by one with a higher priority and explaining the situation and the reason for the overruling.

\footnotetext{
${ }^{3}$ Known as the Contracting Officer's Technical Representative (COTR) in other Agencies.
} 
The 15th International Symposium of the International Council on Systems Engineering (INCOSE),

Determination of the areas of concern for the major stakeholder. The major areas of concern raised by NASA Project Management, the major stake-holder, are summarized as follows:

- The MSOCC Requirements for a switch might not be completely supported by a commercial off-the-shelf (COTS) switch.

- The estimated cost of the MCSS necessitated that the NASA procurement system be used instead of the SEAS Procurement System to procure a COTS switch. This would delay the procurement.

- The transition of the ST modification would add complexity to the installation of the MCSS.

- The DOCS software had to accommodate the transition to the MCSS without changes.

Identification of the operational scenarios for the MCSS. The performance requirements for the MCSS were to be based on a number of MSOCC operational concept scenarios categorized as Normal and Contingency modes.

The Normal Mode scenarios were:

- Pass Support Scenarios - in which LEO data were ingested and processed.

- Simulation and Test Scenarios - which were used for training.

The Contingency Mode scenarios were:

- Trouble-shooting scenarios.

- DOCS unavailable scenarios.

- NASCOM line pre-emption scenarios.

Determination of requirements for the MCSS. The MCSS functional requirements were directly obtained or derived from the MSOCC Functional Requirements Document (FRD), based on the operations scenarios as well as the analysis of information obtained during interviews with MSOCC operations and maintenance personnel and other stakeholders. The non-functional requirements were those that ensured that the equipment power needs were within the capability of the building supply and others inherited from GSFC generic equipment specifications. One set of these generic equipment specifications required that the MSCSS would be installed in standard Electronic Industry Association 19" racks. The paint colour for the racks was inherited when the grounding requirement was inherited from the Spacecraft Tracking and Data Network (STDN) equipment specifications-7. However, there was to be no requirement on the colour of the front panels of the MCSS to be installed in the racks. It was pointed out to the ATR that inheriting the colour requirement for the racks:

- added unnecessary cost of the system since COTS racks were not painted in the specified colour; and also

- made little sense since there was no requirement on the colour of the front panels of the equipment to be contained in the racks.

The ATR however would not waive the inherited requirement for the colour of the racks.

As the requirements were collected they were grouped into the following categories. 
The 15th International Symposium of the International Council on Systems Engineering (INCOSE),

- Functional and non-functional. These were the requirements for the data stream throughputs, the ways of controlling the operation of the switch, and the requirements pertaining to the location of the equipment. The determination of the functional requirements was straight forward. The signal requirements for the replacement MCSS were to be compatible with, and improve on, the then current NASCOM switch in terms of numbers of inputs and outputs, data rates, data and synchronising pulse electrical voltages and currents as discussed above. The determination of the command and control requirements were more complicated because different stakeholders had different ideas of how the replacement switch would be controlled and the number of control points.

- Transition. The transition requirements were the major problem, because the prime directive was "no loss of data". The MSOCC was receiving data from a number of LEO satellites, and either the NASCOM switch or the MCSS had to be active and route the data to the appropriate destinations as and when data were received, and there were no long periods of time when no data was being received from at least one satellite. To compound the problem, the MSOCC was full of equipment and there was no free space to install a complete MCSS without first removing the NASCOM switch. Moreover, in building temporary bypasses, the length of the cables to be used for the signals was limited, since the usable clock rate decreased as the length of the cable increased.

Pre-SRR architecting. Once the requirements for the data stream throughputs had been identified, we performed some risk management to alleviate the major stakeholder (NASA Project Management) concerns. As a result of that activity, we determined that COTS data switches could meet the data stream throughput requirements, but they might not meet the control requirements of all the stakeholders. Moreover, the COTS switches were supplied in modules that would have to be integrated either by the COTS vendor, or by a MSCOCC support contractor. This finding was communicated to the ATR.

Identification of candidate switch architectures. Three alternative implementation architectures were identified. They were:

- Buy a COTS switching system.

- Build a new switching system.

- Build/Buy combinations. Buy some COTS elements and build add-ons to interface to the existing equipment in the MSOCC.

The advantages and disadvantages of the three alternatives were investigated and are summarized in Table 1.

The relatively low cost and implementation risk of the COTS Switches as compared to the design and development of a custom product was examined and a decision was made to go with a COTS product for the switching subsystem. Cost was an important attribute with the need to keep the cost of purchased items within the SEAS procurement limit. However, this decision limited the range of the command and control requirements to the functionality of the COTS switches, and, as the switching requirements were of higher priority than the control requirements, the limitation was accepted.

This decision eliminated a major risk, and determined that the requirements for the replacement switch would be feasible, and achievable within budget. As a result of this decision, the recommended approach was the Build/Buy combination which would 
The 15th International Symposium of the International Council on Systems Engineering (INCOSE),

\begin{tabular}{|c|c|c|}
\hline Option & Advantages & Disadvantages \\
\hline \multirow[t]{2}{*}{$\begin{array}{l}\text { Buy a COTS } \\
\text { product }\end{array}$} & Low developmental risk & $\begin{array}{l}\text { Provided COTS capability would not be } \\
\text { exactly equal to the MSCC requirements }\end{array}$ \\
\hline & $\begin{array}{l}\text { Proven COTS switch } \\
\text { technology }\end{array}$ & $\begin{array}{l}\text { Large amount of customization would be } \\
\text { costly }\end{array}$ \\
\hline \multirow[t]{3}{*}{$\begin{array}{l}\text { Build a new } \\
\text { MCSS }\end{array}$} & $\begin{array}{l}\text { Built to exact MCSS } \\
\text { requirements }\end{array}$ & High developmental risk \\
\hline & $\begin{array}{l}\text { Could be built in a way to } \\
\text { facilitate transition of the } \\
\text { MCSS into the MSOCC }\end{array}$ & $\begin{array}{l}\text { Long lead time } \\
\text { High cost for lifetime support }\end{array}$ \\
\hline & & May have to customize control software \\
\hline $\begin{array}{l}\text { Build/Buy } \\
\text { combination }\end{array}$ & $\begin{array}{l}\text { Uses proven COTS technology } \\
\text { for the data switching } \\
\text { subsystem } \\
\text { Could be built in a way to } \\
\text { facilitate transition of the } \\
\text { MCSS into the MSOCC } \\
\text { Minimal developmental risk }\end{array}$ & $\begin{array}{l}\text { Customization of software, either in the } \\
\text { DOCS or in the MCSS }\end{array}$ \\
\hline
\end{tabular}

Table 1 The advantages and disadvantages of the three alternatives.

purchase the COTS switch components and other standard items such as the control terminals, and build small subsystems as required to interface the MCSS to the MSOCC. This choice also had the advantage of alleviating a major stakeholder concern, namely that the cost of the purchased components became low enough that the SEAS procurement process could be employed.

Identification of candidate transition approaches. The next step was to develop candidate alternative transition plans that met the constraints and the prime directive, based on our knowledge of the MSOCC and its operations. We recognized that the prime directive of "no loss of data" did not equate to "no down time". There were short periods of time when no data were being received and these times could be determined in advance. Thus each candidate transition approach could incorporate some down time when data sources and sinks were being rerouted to the replacement MCSS. We met with the stakeholders again at their convenience and discussed each transition approach and their advantages and disadvantages. We then surveyed the stakeholders as to their preferences. Since the preferences of the stakeholders in the system, being a plurality, had different impacts, we identified a weighting scheme for prioritizing the preferences of the stakeholders. The survey requesting that the evaluation criteria be ranked by the respondent, both in the order of relative importance (i.e. which was more important than the other on a scale of 1-8, with 8 being the most important) and standalone importance (how important each was in itself on a scale of 1-10) was sent to the MSOCC operations, maintenance and engineering personnel. The survey results were summarized in both categories by adding the individual scores and dividing by the number of survey forms returned. No attempt was made to remind people to return the survey forms on the assumption that if they didn't return the completed survey form, they weren't interested in the matter. This assumption had one unfortunate effect. There was one late response to 
The 15th International Symposium of the International Council on Systems Engineering (INCOSE),

the survey by a curmudgeon who provided negative responses to all three transition approaches, with no additional positive suggestions. Since his responses arrived some time after we had finished processing the timely responses, they were given all the consideration they deserved.

Determination of test methodology for MCSS upgrade. The test methodology determined the test and evaluation requirements. It was integrated into the transition plan to promote a low-risk approach based on the following goals:

a. To verify that the MCSS was functional before transition to the MSOCC.

b. To minimize the impact to the MSOCC.

c. To ensure one tested change to the MSOCC at any one time during switch system transition.

d. To establish transition stage milestones to provide both a measurement of progress and contingency fallback positions.

e. To always have two control hosts for each switching system at any time during the transition (i.e., DOCS for the current switches, and the new Local Control Terminals for the MCSS).

f. To minimize interdependencies between the different transition activities.

The Systems Requirements Review. We combined all the relevant information into a single draft requirements document and an overview SRR presentation. The requirements documents contained:

- the performance requirements for the replacement switch;

- the candidate MCSS architectures;

- the recommended architecture and the rationale for the recommendation;

- the transition requirements;

- the alternative transition plans;

- the transition survey results; and

- the recommendation for transition from the existing NASCOM switch to the replacement MCSS, based on the weighted evaluation criteria.

We circulated the draft requirements document (NASA/GSFC, 1989a) before the SRR for stakeholder review and then presented the SRR (NASA/GSFC, 1989b). After summarizing the requirements, the candidate alternatives for transition, and the evaluation criteria for selecting the recommended approach, we presented the recommended transition plan as a high-level sequence of activities (process). Consensus on all requirements and the transition plan was achieved.

\section{Analysis and commentary}

Consider the following aspects of the case description.

- Stakeholder involvement in requirements elicitation

- Process architecting

- The object-orientated approach

- Completeness of the SRR as a result of applying lessons learnt from other people's experiences

- Supply chain requirements 
The 15th International Symposium of the International Council on Systems Engineering (INCOSE),

- Decision makers need the authority to make the decisions

- Organizational roles.

Stakeholder involvement in requirements elicitation. There were two categories of requirements for the MCSS. The first dealt with the performance and control of the MCSS, the second dealt with the transition process for upgrading the NASCOM switch into the MCSS in an MSOCC that didn't have space to install the entire MCSS in one go. Consequently, a staged upgrade would have to be planned, and designed in such as manner to ensure that the downtime of a switch would not impact the prime directive and satisfy all the stakeholders.

The stakeholders were involved in both aspects of the requirements elicitation process (Kotonya and Summerville, 2000). The initial set of stakeholders was identified by determining who was involved in the operations and maintenance of the MSOCC. A classic soft-system intervention approach was used (Checkland, 1993) to determine both sets of requirements by discussing the needs of the stakeholders based on the operations scenarios. Opening the communications channels and providing an understanding of the needs of the various stakeholders usually immediately resolved any issues.

Process-architecting. Theoretical systems engineering teaches that the steps in the process are:

1. Develop a complete set of requirements.

2. Develop alternative candidate designs that meet the requirements,

3. Develop the evaluation criteria to be used to select the optimal candidate.

4. Select the optimal candidate.

5. Build the system.

Had we followed the standard process, we would have had to elicit, elucidate, and document requirements for the MCSS that could have been met by all three candidate architectures. The command and control requirements would have been interesting and probably too expensive to implement since the stakeholders had no idea of the cost of implementing their requirements. After some reflection, the elicitation and elucidation process was architected (Kasser, 2005) so as to employ a soft-system interventionist methodology (Avison and Fitzgerald, 2003) coupled with an object-oriented approach, which very quickly identified the build/buy approach as the optimal one.

The object-oriented approach. The object-oriented approach to requirements engineering considers that a requirement is more than just the imperative statement of the form "the system shall something" (Kasser, 2003). The object-oriented approach not only includes the concept of inheritance but also draws its roots from Total Quality Management (TQM) and contains attributes of the requirements that affect the production process (Kasser, 1995). Thus, at the same time as the product (functional and nonfunctional) requirements were documented, their attributes in the process dimension, namely risk, priority, and cost, were also discussed with the ATR.

The initial set of functional requirements for the MCSS was inherited from the existing NASCOM Switch; the non-functional requirements from the generic requirements for equipment installed at the GSFC. These requirements were then tailored (improved) by considering the stakeholder responses to the three critical questions:

- What is good about the current system? 
The 15th International Symposium of the International Council on Systems Engineering (INCOSE),

- What is bad about the current system?

- What would you change, and why?

The weighting of the responses was such that responses from those who interacted with the NASCOM Switch were allocated a higher priority than the responses from those who managed it.

The data switching requirements had the highest priority. By considering the cost and risk attributes at the same time as the performance attribute, risk management was built into the requirement elicitation and elucidation process. Thus the implementation risk in building the data switching subsystem of the MCSS led to the early decision to purchase COTS products for that subsystem. Cost was an important attribute with the need to keep the cost of purchased items within the SEAS procurement limit. However, the decision to purchase a COTS switch limited the range of the command and control requirements to the functionality provided by the COTS switches, and, as the switching requirements were of higher priority than the control requirements, the limitation was accepted by the ATR and other stakeholders.

Completeness of the SRR. Milestone Reviews were held at the GSFC in the form of presentations to stakeholders. Feedback, suggestions, omissions, and comments took the form of Review Item Discrepancies (RID) in which written comments were made about some perceived deficiency in the requirements. These RIDs were tracked, assessed, and reported on, at the following milestone review.

(Kasser and Schermerhorn, 1994a) discussed two metrics for reviews. One metric for reviews was the number of RIDs generated by a review. Since reviews with the same name (i.e. SRR) for different projects have different levels of complexity depending on the size of the system being reviewed, comparing numbers between different projects may not be useful. However, measuring the number of RIDs does provide a metric for the degree of customer involvement in the process. The larger the number of RIDs generated, the lower the involvement of the customers, because the RIDs would have been preempted by the pre-review dialogue.

The second metric was the amount of rework to be performed following the review, or the amount of work that has to be scrapped and replaced. In percentage terms, the metric can be used to compare the effectiveness of the Systems Engineering Team across different projects. The MCSS SRR did not generate any RIDs so in this case there was no scrap and no rework was needed.

What did we do correctly? We applied TQM to Systems Engineering. As (NASA, 1992) stated "TQM is the application of Systems Engineering to the work environment". Thus as we started the task, we researched factors that determined project successes and failures, namely looked at the lessons learned from experience and the literature and published the results in (Kasser and Schermerhorn, 1994b) and (Kasser, 1995). We speedily identified that clear and concise communications were the key to the success of a requirements elicitation and elucidation project. Later we also located a survey of quality implementation in 100 different companies (BBP, 1990, 1991) which showed that the single most important factor for success was a common vision of:

- what the system was supposed to do

- who the customer was, and 
The 15th International Symposium of the International Council on Systems Engineering (INCOSE),

- the implementation plan for achieving the goals.

We determined that our direct customer was the ATR, but there were important indirect customers, namely the stakeholders. Our identification of the customers and their concerns, the communication of the vision of what the MCSS was supposed to do and the implementation or transition plan were the major factors in achieving an outstanding SRR which resulted in no RIDs being generated, something which was unprecedented at the time ${ }^{4}$.

Alas the follow-on implementation activities to construct the MCSS fell into the grey area of overlap between the SEAS and NMOS contracts. The NMOS contractor was perceived as being cheaper than the SEAS contractor, so the implementation task was awarded to NMOS. This tends to reinforce the concept of not completing the task in US Government contracts so as to obtain follow-on work. An example of this situation was Kirtland Air Force Base's sole source follow-on award to CTA Inc. (CBD, 1995). The reasons for that sole-source follow-on contract were stated in the Commerce Business Daily as being lack of documentation and insufficient existing data for a different contractor to economically take over the contract from the incumbent.

Supply-chain requirements. The transition requirements fell into the categories of supply chain requirements or constraints. They were not concerned with the functionality of the MCSS, they were concerned with the supply (installation) of the equipment to the MSOCC. Supply chain requirements tend to be overlooked in government procurements. Legends abound about the delivery of equipment to submarines, in which the equipment is constructed in such a manner as to require a hole to be cut in the hull to take the equipment aboard; or the failure to consider launch vibration conditions when building spacecraft. In the object-oriented paradigm, the number of 'forgotten' requirements of this type can be reduced by inheriting them from the class of equipment. Thus for example, spacecraft can inherit launch vibration, and thermal vacuum requirements. The same concept of inheritance can be applied to classes of non-functional requirements such as environmental requirements.

\section{Decision makers need authority to make the decisions.}

When the inherited requirement for the colour of the racks was identified as adding cost and being illogical, the ATR made an informed decision to keep the requirement. The appeal to the ATR to waive the requirements for painting the 19 inch racks to the STDN-7 colour was an appeal to the wrong person. We assumed the ATR had the authority and would be willing to waive the requirement. We should have identified who had the authority and made sure they were included in the discussions on the colour requirement and the effect of not waiving the requirement on cost and schedule. By the time we realised this, it was too late to do so without appearing to overrule the ATR, so we didn't, and just noted the lesson learnt.

Organizational roles. During the course of this task, we thought of the systems engineer as the project expert who ensures that the process is optimally planned and implemented during the course of the project life cycle (Kasser and Schermerhorn, 1994b). Thus not

\footnotetext{
${ }^{4}$ Since RIDs can also be considered as metrics for the completeness of a review, we either did an outstanding job, or nobody cared about the MCSS.
} 
The 15th International Symposium of the International Council on Systems Engineering (INCOSE),

only did we perform the organizational role of systems engineering in determining the requirements for the MCSS, we also performed the organizational role of systems architecting (Maier and Rechtin, 2000) in developing the candidate architectures for the MCSS, and the role of process architecting (Kasser, 2005) in developing the transition plan.

\section{Lessons learned}

The lessons learned on this project are summarized as follows.

- Stakeholder participation is critical to the success of any project especially when a plurality is involved (Kotonya and Summerville, 2000), (Flood and Jackson, 1991). Everyone gets their needs addressed, and if they are not met, they understand the reasons why they were not met. This was ensured by involving the stakeholders in determining both the requirements for the MCSS and how the transition from the NASCOM switch to the MCSS would occur

- Decisions should be discussed with those who have the authority to make the decisions and are willing to do so.

- In US government contracts, always leave something undone. In this instance it was don't solve the entire problem, or the competing (cheaper) contractor will get the follow on (implementation) task.

\section{Summary}

In summary, the MSOCC Switch upgrade task was an exercise in requirements elicitation. The functional requirements were relatively simple to identify. The control requirements were more difficult since there was a plurality of stakeholder needs. However, the most important requirements were the supply chain requirements pertaining to the actual transition from the NASCOM switch to its replacement MCSS rather than the performance of the MCSS. The soft system intervention approach was crucial to the success of the task.

\section{Conclusions}

The following conclusions can be drawn from this case study:

- While the performance requirements are mandatory, sometimes the supply chain and process requirements are just as, or even more, critical.

- The context in which the system is being implemented must be considered when determining the system requirements.

- Systems engineers involved in the elicitation and elucidation of requirements need to add soft systems methodologies to their toolboxes.

\section{Acronyms}

AP

ATR

ASPC

CBD

$\mathrm{CMF}$
Application Processor

Associate Technical Representative

Attached Shuttle Payload Center

Commerce Business Daily

Command Management Facility 
The 15th International Symposium of the International Council on Systems Engineering (INCOSE),

COTR Contracting officer's technical representative

COTS Commercial off-the- shelf

CPAF Cost plus award fee

DOCS Data Operations Control

DSN Deep Space Network

DTE Data Terminal Equipment

FRD Functional Requirements Document

GSFC Goddard Space Flight Center

INCOSE International Council on Systems Engineering

HRS

LEO

MCSS MSOCC Communication Switching System

MPT Mission Planning Terminal

MSOCC Multiple-Satellite Operations Control Center

NASA National Aeronautics and Space Agency

NMOS Network Maintenance and Operations Support

PI Principal Investigator

RID Review Item Discrepancy

RUPS Recorder Utility Processors

SEAS Systems Engineering and Analysis Support

SRR System Requirements Review

STDN Spacecraft Tracking and Data Network

TPOCC Transportable Payload Operation Control Center

\section{References}

Avison, D. and Fitzgerald, G., Information Systems Development: Methodologies, Techniques and Tools, McGraw-Hill Education (UK), Maidenhead, 2003.

BBP, Excellence Achieved, Customer Service, Blueprints for Action from 50 Leading Companies, Bureau of Business Practices, 1990.

BBP, Quality Excellence Achieved, Quality Assurance, Blueprints for Action from 50 Leading Companies, Bureau of Business Practices, 1991.

CBD, "Kirtland Air Force Base's proposed sole source follow-on award to CTA Inc., Commerce Business Daily, April 18, 1995", (1995).

Checkland, P., Systems Thinking, Systems Practice, vol. Chichester, John Wiley \& Sons, 1993.

Flood, R. L. and Jackson, M. C., Creative Problem Solving, Wiley, 1991.

Kasser, J. E., Applying Total Quality Management to Systems Engineering, Artech House, Boston, 1995.

Kasser, J. E., "Object-Oriented Requirements Engineering and Management ." The Systems Engineering Test and Evaluation (SETE) Conference, Canberra, 2003.

Kasser, J. E., "Introducing the Role of Process Architecting", The 15th International Symposium of the International Council on Systems Engineering (INCOSE), Rochester, New York, 2005.

Kasser, J. E. and Schermerhorn, R., "Determining Metrics for Systems Engineering", The 4th Annual International Symposium of the NCOSE, San Jose, CA, 1994a.

Kasser, J. E. and Schermerhorn, R., "Gaining the Competitive Edge through Effective 
The 15th International Symposium of the International Council on Systems Engineering (INCOSE),

Systems Engineering", The 4th Annual International Symposium of the NCOSE, San Jose, CA, 1994.

Kotonya, G. and Summerville, I., Requirements Engineering processes and techniques, John Wiley \& Sons, Chichester, 2000.

Maier, M. K. and Rechtin, E., The Art of Systems Architecting, CRC Press, 2000.

NASA, NASA Systems Engineering Handbook, draft, September 1992, 1992.

NASA/GSFC, Multi-Satellite Operations Control Center (MSOCC) Communications Switching System (MCSS) System Requirements Document, 511-4SRD/0489, November 1989, 1989a.

NASA/GSFC, Multi-Satellite Operations Control Center (MSOCC) Communications Switching System (MCSS) System Requirements Review, December 1989, 1989b.

\section{Author's biographies}

Joseph Kasser has been a practising systems engineer for 30 years. He is the author of "Applying Total Quality Management to Systems Engineering" and many INCOSE symposia papers. He holds a Doctor of Science in Engineering Management from The George Washington University, and is a Certified Manager. He is the DSTO Associate Research Professor at the Systems Engineering and Evaluation Centre at the University of South Australia. He performs research into the nature of systems engineering and the properties of object-oriented requirements while managing the customised section of the Master of Project Management degree for the Australian Defence Materiel Organisation. $\mathrm{He}$ is a recipient of NASA's Manned Space Flight Awareness Award (Silver Snoopy) for quality and technical excellence for performing and directing systems engineering.

Chandru Mirchandani Received his ME in Electrical Engineering from Rensselaer Polytechnic Institute, Troy NY, and an MS in Reliability \& Systems Engineering at the University of Maryland, College Park, MD. Worked as a Research and Development Engineer at the Monolithic Devices Laboratory, Hewlett-Packard, Colorado Springs, Faculty Member in the Department of Electronics and Physics at the Institute of Technological Studies, Sri Lanka. Working for Lockheed-Martin Space Operations, he supported Goddard Space Flight Center, as a Senior Staff Engineer in the research, development, design and integration of VLSI-based telemetry systems using state-of-theart technologies in ASIC design, PCB design and data transfer \& storage. Currently, with Lockheed-Martin Transportation and Security Solutions, as Lead Reliability, Maintainability and Availability Engineer on the En-Route Traffic Management (ERAM) System, a safety critical Air Traffic Control program for the FAA. Admitted to the Doctoral program at George Washington University, he is pursuing a D.Sc. in Systems Engineering. Interests include the research, design and model development of systems based on reliability, performance and cost; fault-tolerant systems; Bayesian processes and decision theory. 